\title{
Early Experience with Local Custom Made Synthetic Bone Construct for the Treatment of Bone Defect
}

\author{
Nazri Mohd Yusof ${ }^{1}$, Harkeerat Singh ${ }^{1}$ \\ ${ }^{1}$ Department of Orthopaedics, Traumatology and Rehabilitation, Kulliyyah of \\ Medicine, International Islamic University Malaysia
}

Presenter: Harkeerat Singh

Introduction: Segmental bone defect can be treated with autologous bone graft and bone transport. Synthetic bone graft has successfully been used for the treatment of metaphyseal fracture or bone cyst. However, their use for the treatment of segmental bone defect has not been establish. Recently, a local company has produced a biodegradable custom made synthetic bone construct phosphate-based materials that is used to treat fractures and bone defect. Materials and Methods: After getting approval from Medical Device Agency, we used SBC to treat segmental bone defect in our patients. A case series review was conducted in these cases. Results: Three patients underwent surgery using SBC as a spacer to fill the defect at the docking site to prevent soft tissue collapse in one patient and as a replacement for bone autologous bone grafting in two patients. In our observation granules resorbtion ranged from $40-80 \%$ by 6 months with no radiological evidence of new bone formation. SBC mixed with bone marrow aspirate failed to harden into a palette. Although no new bone formation was seen at the docking site, insertion of SBC prevents soft tissue invagination and reduced the need of a second surgery to remove other conventional spacers. Conclusion: SBC may be used as a spacer to prevent soft tissue interposition and skin invagination in bone transport. However mixing it with bone marrow aspirate resulted in failure to form beads and does not form bone. 